INTERNATIONAL JOURNAL OF SCIENTIFIC RESEARCH

\title{
A CASE REPORT ON SYSTEMIC LUPUS ERYTHEMATOSUS PRESENTING WITH ACUTE ABDOMINAL PAIN AND THE ROLE OF IMAGING IN LUPUS MESENTERIC VASCULITIS
}

\section{Radiodiagnosis \\ Dr. Vemuri Naga Varaprasad}

Dr. Songa Ramya*
MD FICR, Chief Radiologist, Global Multispeciality Hospital, Vijayawada, Andhra Pradesh, India

MD, Department of Radiology, Global Multispecialty Hospital, Vijayawada, Andhra Pradesh, India. ${ }^{*}$ Corresponding Author

\section{ABSTRACT}

Acute abdominal pain is seen during the active phase in around 8-40\% of SLE patients. Lupus mesenteric vasculitis (LMV) is one of the common causes of acute abdomen in SLE patients; however, it is an uncommon condition with a global prevalence of $0.2-9.7 \%$. Clinical symptoms and laboratory parameters are non-specific in the diagnosis of LMV. Nevertheless, radiological investigations like ultrasonography and computed tomography play a role in narrowing down the differential diagnosis and excluding other non SLE causes of acute pain abdomen and aids in the management of the patient.

\section{KEYWORDS}

SLE, Lupus Mesenteric Vasculitis, Acute pain abdomen

\section{INTRODUCTION:}

Lupus Mesenteric Vasculitis (LMV) is also known as lupus enteritis, mesenteric vasculitis, or acute gastrointestinal syndrome[1]. LMV is one of the common causes of acute pain abdomen in SLE patients with variable geographic prevalence, i.e. high prevalence was seen in Asia (2.2\%-9.7\% ) and a lower prevalence rate of $0.9 \%$ in America. LMV needs to be differentiated from other SLE, and non-SLE related causes of acute pain abdomen as this condition may escalate from simple bowel wall thickening to life-threatening complications without prompt immunosuppressive therapy[2].

Clinical symptoms and laboratory parameters are non-specific in the diagnosis of LMV. Radiological investigations like ultrasonography (USG) and computed tomography (CT) aids in narrowing the differential diagnosis and excluding other causes of acute pain abdomen. Contrast-enhanced CT (CECT) allows visualising bowel wall status, abdominal vasculature for thrombotic occlusion and associated complications. Biopsy gives conclusive evidence.

\section{Case report:}

A 23 years old female patient came with chief complaints of acute pain abdomen and diarrhoea with no similar complaints in the past. She was diagnosed with SLE for 6 years.

Laboratory parameters: At the time of admission, the patient had anaemia (Hb-7.9gm \%), decreased leucocyte count-3,800cells $/ \mathrm{ml}$, elevated ESR-90mm $/ \mathrm{hr}$, elevated Serum Creatinine and Blood Urea levels- 2 and $4 \mathrm{mg} / \mathrm{dl}$ respectively, overt proteinuria with urine protein: creatinine ratio-1.7. Anti ds DNA antibody -positive, antinuclear antibody-strongly positive and decreased $\mathrm{C} 3$ and $\mathrm{C} 4$ levels.

Ultrasonography revealed diffuse symmetrical hypoechoic bowel wall thickening involving small and large bowel loops with loss of gut signature and mild ascites. (figure: 1 )

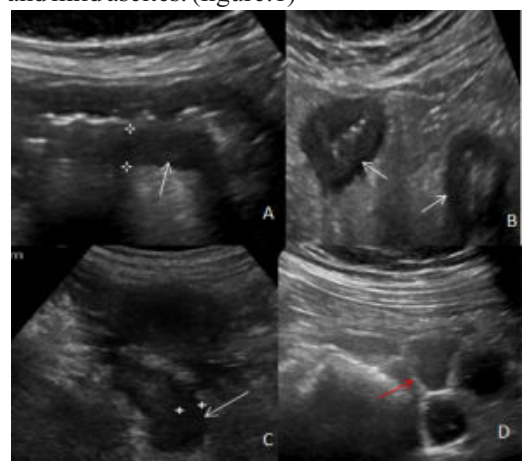

Figure:1USG revealed long segment symmetrical thickening (white arrows)of small bowel loops(A\&B) and rectum(C) with loss of gut signature, follow up USG revealed normal thickness of bowel wall(Dred arrow)
$C E C T$ revealed long segment circumferential symmetrical thickening of small bowel loops, descending, sigmoid colon and rectum with normal bowel loops in between. On contrast administration, bowel wall thickening showed grey target pattern of enhancement with adjacent fat stranding and mild ascites. Mesenteric vessels are mildly engorged(combs sign positive) with no evidence of thrombus. (figure:2)

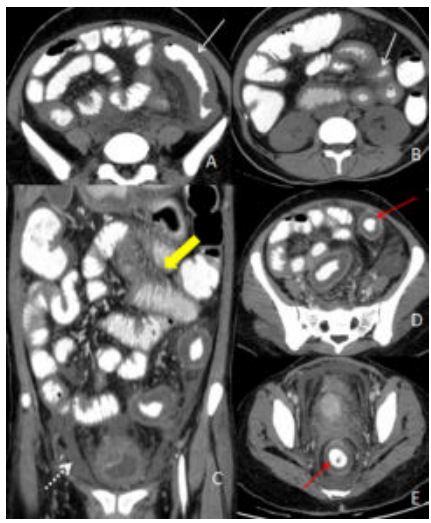

Figure:2 CECT abdomen showing diffuse symmetrical circumferential thickening of small bowel loops (b-white arrow), sigmoid colon (a-white arrow), with gray target pattern of enhancement (d,e- red arrows), vascular engorgement (c-yellow bold arrow) and ascites (c- white dotted arrow).

No other causes of acute abdominal pain were identified both in USG and CECT abdomen.

These features were in favour of the diagnosis of lupus mesenteric vasculitis and our diagnosis was further supported by the fact that patient recovered in 3days after the administration of high dose intravenous steroid therapy (Methylprednisolone $1 \mathrm{mg} / \mathrm{kg} / \mathrm{day}$ ).

Followup USG after 3 weeks revealed significant decrease in bowel wall thickening. Though CT is preferred over USG in diagnosing bowel wall pathology, since patient had multiple CT scans so far, USG was done to prevent further radiation exposure(figure:1D).

\section{DISCUSSION:}

Acute abdominal pain is seen during the active phase in around $8-40 \%$ of SLE patients and among them, around $29 \%$ to $65 \%$ are due to LMV[2]. Multiple conditions can cause acute abdominal pain, including both SLE, associated and non-SLE conditions, listed in the table:1.[3]. LMV is one of the common causes seen among SLE patients with acute abdominal pain. A wide spectrum of clinical features is reported from simple non-specific abdominal pain, bloating and loose stools to life-threatening complications like necrosis, perforation and haemorrhage [4]. 
Table:1 Causes of acute abdominal pain in SLE patients

\begin{tabular}{|l|l|}
\hline SLE related & Non SLE related \\
\hline Lupus mesenteric vasculitis & Peptic ulcers \\
\hline Protein-losing enteropathy & Appendicitis \\
\hline Pseudo obstruction & Calculus cholecystitis \\
\hline Acute pancreatitis & Pancreatitis \\
\hline Acalculus cholecystitis & Ovarian pathology \\
\hline $\begin{array}{l}\text { Mesenteric thrombus (anti } \\
\text { phospholipid antibody syndrome) }\end{array}$ & Diverticulitis \\
\hline $\begin{array}{l}\text { Drug induced- NSIADS, } \\
\text { immunosuppresants (CMV colitis) }\end{array}$ & Adhesions, intestinal occlusion \\
\hline & Pyelonephritis \\
\hline
\end{tabular}

Leucopenia and anti-endothelial cell antibodies (AECAs) are the only parameters that correlate with the occurrence of lupus vasculitis[4] as seen in our patient. Other parameters like Systemic Lupus Disease Activity Index (SLEDAI), autoantibodies (anti-phospholipid antibody, lupus anticoagulant, anti-cardiolipin antibody, and anti- $\beta 2-$ glycoprotein antibody) and other laboratory indices though elevated in SLE, do not correlate with the occurrence of lupus vasculitis $[1,4]$.

Vasculitis in SLE involve almost any part of the GI tract from the oesophagus to the rectum but predominantly affect the distribution of the superior mesenteric artery and rarely involves rectum [5]. Before a diagnosis of lupus vasculitis is made, other causes of vascular insufficiency should be excluded like immunotherapy associated cytomegalovirus (CMV) and other infections, leading to colitis. Thrombosis of mesenteric vessels associated with the antiphospholipid syndrome can give rise to visceral or mesenteric ischemia and bowel infarction

\section{Pathogenesis:}

Inflammatory vasculitis and mesenteric thrombosis are proposed pathogenic mechanisms of LMV. Inflammatory vasculitis mediated by immune complex deposition and thrombosis of the intestinal vessels secondary to circulating anti-phospholipid antibodies. [1,4,6]. Both types of microvasculopathy can activate each other reciprocally, resulting in worsening cascades of vasculitis and thrombosis[1]. Patient with lupus have anti-phospholipid antibody syndrome and are at risk for the development of mesenteric arterial or venous thrombosis in $27-42 \%$ of cases[7].

\section{Imaging findings:}

Accurate diagnosis of LMV is critical to allow prompt treatment and avoid unnecessary surgical intervention. As the clinical symptoms and laboratory parameters are non-specific, and bowel specimens are not always available, the diagnosis of LMV relies on imaging.

Double-contrast radiography shows irregular thickening and projection of folds in multiple segments of the duodenum and the terminal ileum accompanied by the "thumbprint" sign suggests ischemic changes[1].

Ultrasonography is also useful for both the diagnosis and follow-up of LMV. Small intestinal wall oedema with loss of gut signature and bowel wall thickening can be visualised under ultrasonography. Similar findings were seen in our patient[1].

Contrast-enhanced CT findings in patients with LMV includes dilated bowel, focal or multisegment bowel wall thickening not confining to a single vascular territory usually involving jejunum and ileal loops rarely involving rectum and colon which is seen in our case. Abnormal bowel wall enhancement which is also called "target sign", mesenteric oedema, stenosis or engorgement of mesenteric vessels which is also called "the comb sign" and ascites. Segmental or multifocal involvement of the small and large bowel loops with intervening normal bowel segments indicates ischemic change, which is almost always indicative of vasculitis $[8,9,10]$ and summarized in table:2.

\section{Table: 2 CECT findings in LMV}

\section{CECT findings in LMV}

\begin{tabular}{|l|ll|}
\hline Bowel wall & $\bullet \begin{array}{l}\text { Dilated bowel loops } \\
\text { Focal or multisegment bowel wall thickening } \\
\text { not confining to a single vascular territory }\end{array}$ \\
\hline Mesentry & $\begin{array}{l}\text { Abnormal bowel wall enhancement which is } \\
\text { ("target sign") }\end{array}$ \\
\hline $\begin{array}{l}\text { Mesenteric oedema, } \\
\text { Stenosis or engorgement of mesenteric vessels } \\
\text { ("the comb sign") }\end{array}$ \\
\hline
\end{tabular}

\begin{tabular}{|l|l|}
\hline Miscellaneous & Ascites. \\
\hline
\end{tabular}

CT also plays a role in the efficiency of treatment in patients treated with high dose iv steroids in follow-up patients. Ko et al. in their study stated a significant decrease in bowel wall thickening in patients with subsiding clinical symptoms[11].

\section{Differential diagnosis:}

Anti-phospholipid antibody syndrome: Secondary aPLA syndrome is seen in $27-42 \%$ of SLE patients with mesenteric arterial or venous thrombosis, in contrast to engorged mesenteric vasculature in LMV[7]. Though segmental bowel wall involvement within a vascular territory is seen in most cases, more than one vessel may be thrombosed, causing multisegmental diffuse bowel wall thickening, ascites, and perforation.

\section{Management:}

The treatment includes high dose intravenous infusion of methylprednisolone or an equivalent agent and complete bowel rest. For patients with recurrent LMV and those who do not have an adequate response to intravenous prednisolone alone, intravenous cyclophosphamide should be initiated. A study by Kim's et al. found that a bowel wall thickness greater than $9 \mathrm{~mm}$ usually indicates the presence of recurrent $L M V$ and should be considered a high-risk factor for recurrence. They suggested that for patients with a high risk of recurrence, immunosuppressive agents should be initiated as early as possible. Prognosis of the patient depends on vascular involvement, early high dose steroid treatment and timely surgical intervention $[1,12]$.

\section{CONCLUSION:}

LMV is one of the common causes of acute abdominal pain in SLE patients and is important to differentiate it from other SLE and nonSLE conditions. Clinical and laboratory investigations are nonspecific. Radiological investigations particularly CECT findings though non-specific can aid to excludes other causes of acute pain abdomen, narrow down the differential diagnosis, in follow up and can predict the prognosis of the patient. Though rectal involvement is uncommon, rectal involvement is seen in our case.

Future scope: Only handful of cases of LMV were reported in literature owing to the need to report more cases. Variable radiological pattern of involvement to be studied to make confident diagnosis of LMV. usefulness of ultrasound foe screening and foillow up in suspected cases of LMV

\section{Conflicts of intertest: None}

\section{REFERENCES:}

1. Tian X-P, Zhang X. Gastrointestinal involvement in systemic lupus erythematosus: insight into pathogenesis, diagnosis and treatment. World J Gastroenterol. 2010 Jun 28;16(24):2971-7.

2. Ju JH, Min J-K, Jung C-K, Oh SN, Kwok S-K, Kang KY, et al. Lupus mesenteric vasculitis can cause acute abdominal pain in patients with SLE. Nat Rev Rheumatol. 2009 May;5(5):273-81.

3. Janssens P, Arnaud L, Galicier L, Mathian A, Hie M, Sene D, et al Lupus enteritis: from clinical findings to therapeutic management. Orphanet J Rare Dis. 2013;8(1):67.

Kwok, et al. Lupus enteritis: clinical characteristics, risk factor for relapse and association with anti-endothelial cell antibody. Lupus. (2007); 16(10), 803-809.

John M. Kirby, Kartik S. Jhaveri, Zeev V. Maizlin, Mehran Midia, Ehsan Haider, Korosh John M. Kirby, Kartik S. Jhaveri, Zeev V. Maizlin, Mehran Midia, Ehsan Haider, Korosh
Khalili. Abdominal manifestations of systemic lupus erythematosus: spectrum of imaging findings. Can Assoc Radiol J. 2009 Jun; 60(3): 121-132.

6. Cervera R, Espinosa G, Cordero A, Oltra MR, Unzurrunzaga A, Rossiñol T, et al Intestinal Involvement Secondary to the Antiphospholipid Syndrome (APS): Clinica and Immunologic Characteristics of 97 Patients: Comparison of Classic and Catastrophic APS1. Seminars in Arthritis and Rheumatism. 2007 Apr;36(5):287-96.

7. Richard M Gore, Marc S Levine. Vascular disorders of the small intestine. In: Text book of gastrointestinal radiology. 4th ed. philadelphia: Elsevier/Academic Press; 2015. p. 841 .

8. C. K. Si-Hoe, C. H. Tang, S. G. Chee, E. K. Teo, H. H. Chng.Abdominal computed tomography in systemic lupus erythematosus.Clin Radiol. 1997 Apr; 52(4): 284-289.

9. Lalani TA, Kanne JP, Hatfield GA, Chen P. Imaging Findings in Systemic Lupus Erythematosus. RadioGraphics. 2004 Jul;24(4):1069-86.

10. C Lee, M Ahn, E Lee, J Shin, Y Cho, H Ha, B Yoo, H Moon. Acute abdominal pain in systemic lupus erythematosus: focus on lupus enteritis (gastrointestinal vasculitis). Ann Rheum Dis. 2002 Jun; 61(6): 547-550.

11. Ko SF, Lee T-Y, Cheng T-T, Ng S-H, Lai H-M, Cheng Y-F, et al. CT findings at lupus mesenteric vasculitis. Acta Radiol. 1997 Jan;38(1):115-20.

12. Kim YG, Ha HK, Nah SS, Lee CK, Moon HB, Yoo B. Acute abdominal pain in systemic lupus erythematosus: factors contributing to recurrence of lupus enteritis. Ann Rheum Dis 2006;65: 1537-1538. 